\title{
Palisaded encapsulated neuroma: an uncommon tumour at uncommon location
}

\section{Anup Kumar Tiwary, Shyam Sundar Chaudhary, Dharmendra Kumar Mishra}

\author{
Department of Dermatology, Venereology and Leprosy, Rajendra Institute of Medical Sciences, Ranchi, India
}

Corresponding author: Dr. Anup Kumar Tiwary, E-mail: anup07tunnu07@gmail.com

\begin{abstract}
Palisaded encapsulated neuroma (PEN) is an uncommon, sporadic, benign, intraneural tumour presenting as an asymptomatic, solitary, sessile, immobile, skin-coloured papule or nodule, commonly affecting the "butterfly area" of face in middle-aged person. Other less commonly involved sites are trunk, shoulder and distal part of extremities. We herein report a 45 years old male presenting with a painless, firm,nodular growth over the posteromedial aspect of right knee which was further confirmed to be the case of PEN on histopathological examination.
\end{abstract}

Key words: Intraneural; Palisaded encapsulated neuroma; Schwannoma

\section{INTRODUCTION}

PEN also known as ‘solitary circumscribed neuroma' is an uncommon, sporadic, cutaneous, benign, intraneural tumor, which usually presents as asymptomatic, solitary or multiple, firm, rubbery, skin-coloured or pink, papule or nodule, commonly localized on the face or close to a muco-cutaneous junction in middle - aged adults with equal sex preponderance [1]. It was first described by Reed et al in 1972 [1]. Being solitary or multiple, it has never been seen to be associated with neurofibromatosis or multiple endocrine neoplasia [2]. Clinically, it is more commonly misdiagnosed with schwannoma, neurofibroma and dermatofibroma so it is imperative to histopathological evaluation in all such cases.

\section{CASE REPORT}

A 45 year old male presented with a single, firm, skin coloured, nodular growth on postero-medial aspect of right knee which began as a papule one year back and gradually increased in size to $2 \mathrm{~cm} \mathrm{x} 1 \mathrm{~cm}$ over one year. Initially it was asymptomatic but in last 3 months mild pain and itching was started. The patient was born of non-consanguineous parents and none of the family members were affected.
On general physical examination, patient was healthy and normal looking. All routine investigations and laboratory parameters were within normal limits and non contributory. Systemic examinations including ophthalmological and neurological examinations were also normal and none of the features of neurofibromatosis were detected. On local cutaneous examination, the lesion was solitary, skin coloured, immobile, firm, rubbery, pedunculated, nodular growth, neither warm nor tender but mildly painful and pruritic (Fig. 1). There was no history of trauma, oozing or bleeding from the lesion. Telangiectasia, ulceration or discharge was also absent. Mucosa, hairs, nails and teeth were found to be normal looking on examination. Regional lymph nodes were not enlarged.

Appendageal tumours, schwannoma and dermatofibroma were kept as provisional diagnosis. Excisional biopsy was then performed and histopathological study was done. HPE demonstrated partially encapsulated, well circumscribed dermal neoplasm that was differentiated towards nerves (Fig. 2). It showed several nerve bundles like structure in the form of fascicles containing interdigitating spindle cells having ill defined palisades of nuclei (Verocay bodies) and in between acellular area (Fig. 3).

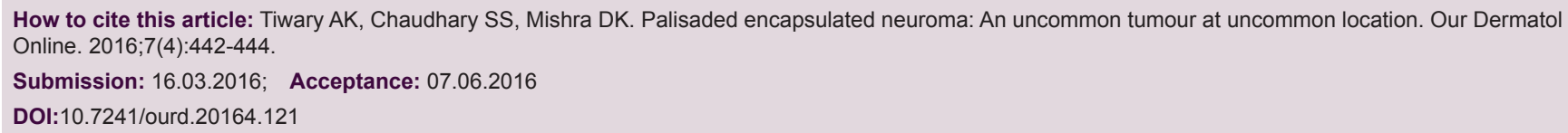




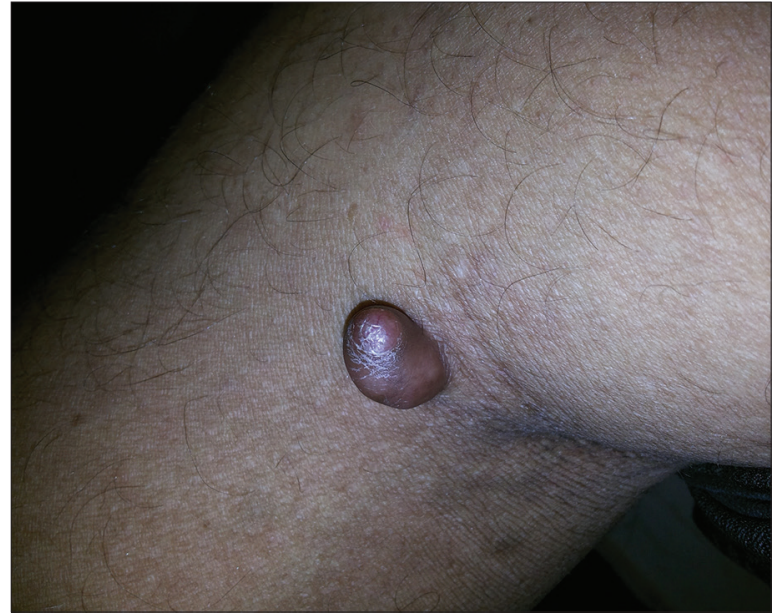

Figure 1: Solitary, firm, skin coloured, nodular growth on posteromedial aspect of right knee.

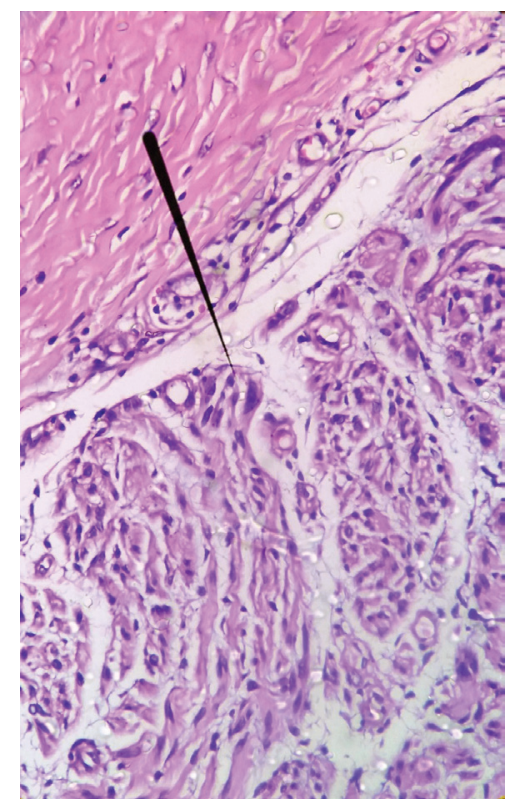

Figure 2: Partially encapsulated, well-circumscribed dermal nodules several nerve bundles like structures in the form of fascicles containing interdigitating spindle shaped shwann cells $(\mathrm{H} \& \mathrm{E}, \times 40)$.

Increased number of small blood vessels were also seen in dermis.

Based on the histopathological findings, diagnosis of PEN was made. After excisional biopsy, wound was closed with mersilk 3-0 suture and patient was advised for follow up to look for any recurrence of the lesion.

\section{DISCUSSION}

$\mathrm{PEN}$ is an intradermal benign, intraneural neoplasm accounting for $25 \%$ of all dermal nerve sheath tumours [3]. It presents as solitary or multiple, firm, rubbery, skin or pink coloured, papule or nodule most

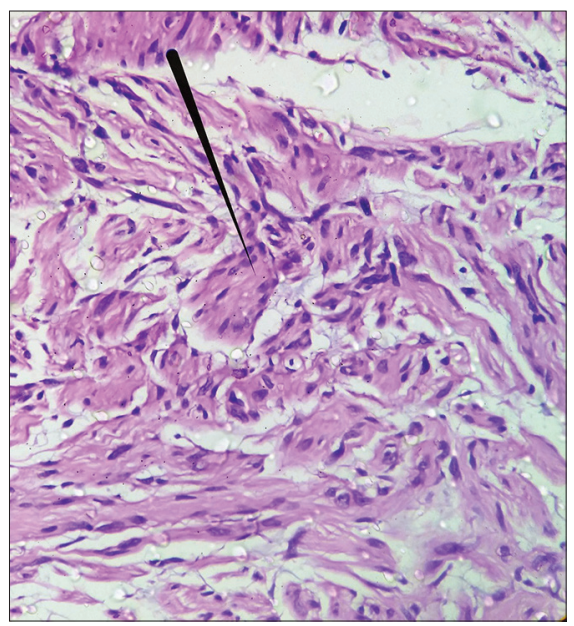

Figure 3: Spindle shaped schwann cells having eosinophilic cytoplasm, pointed and wavy basophilic nuclei and palisading of nuclei and acellular reticular fibres in between these nuclei forming 'verocay bodies' (H\&E, $\times 100)$.

commonly involving face in $90 \%$ of cases. Other less common sites of predilection are trunk, shoulder, arm, hand, foot, oral mucosa, nasal fossa and glans penis [3-5]. There is no sex predilection and usually affects 30-60years age group. Its size usually ranges from $2 \mathrm{~mm}$ to $6 \mathrm{~mm}$ and gradually enlarges over the time. Over the time traumatic ulceration may occur as a secondary complication but haemorrhage and necrosis has not been reported and telangiectasia is minimal or absent. No hairs are seen to come out of the surface.

PEN is supposed to be caused by reactive hyperplasia or hamartomatous growth of schwann cells and axons causing bulbous expansion of nerves but triggering factors are yet to be known [6]. Clinically it is closely simulated by schwannoma, neurofibroma, dermatofibroma, appendageal tumours of skin, epidermal cyst, basal cell carcinoma and intradermal naevi $[1,4,7]$. Relatively, very lower occurrence of telengienctasia and ulceration helps in ruling out the diagnosis of basal cell carcinoma [4]. Neurofibroma, schwannoma and intradermal naevi simulate the clinical picture but can be differentiated on HPE $[1,4]$.

On HPE, it is seen as intradermal nodule composed of encapsulated interlacing distinct fascicles containing spindle cells and separated by artifactual clefts [8]. The tumour has fine capsule or is partially encapsulated because it doesn't include epidermis.

The capsule contains epithelial membrane antigen (EMA) positive flattened, elongated perineural cells [7]. The tumour cells include schwann cells and axons. The schwann cells have eosinophilic cytoplasm, 
pointed and wavy basophilic nuclei and palisading of nuclei may be seen in few fascicles. The palisaded nuclei and acellular reticular fibres in between these nuclei form 'verocay bodies' but are ill-defined usually $[7,5]$. These verocay bodies are more distinct and consistent finding in schwannoma rather than in PEN [5]. Apart from the nodular pattern of intradermal fascicles, other less common patterns have also been reported like plexiform, epithelioid, multinodular, fungating and vascular [9].

On immunohistochemical analysis, capsule stains for EMA, and schwann cells stain for S-100, collagen type IV and vimentin. Axons stain with neuron specific enolase (NSE) and antibodies against neurofilaments.

Histopathologically the close differential diagnoses are schwannoma, traumatic neuroma, neurofibroma, leiomyoma. Usually Schwannoma is located subcutaneously having Antoni - A and Antoni - B areas which are rich in varocay bodies and lack axons [5]. Neurofibroma lacks capsule, rich in mucopolysaccharide ground substance and have fewer axons [8]. Traumatic neuroma closely mimcks the histopathological picture but can be differentiated by the presence of inflammatory cells and scarring. The presence of smooth muscle cells and positive staining for desmin and vimentin clinches the diagnosis of leiomyoma.

Treatment of choice is complete excision and chance of recurrence is very less even after incomplete excision $[4,9]$.

\section{CONCLUSION}

As Palisaded encapsulated neuroma can easily be misdiagnosed with other neural and soft tissue tumours, histopathological evaluation is imperative to confirm the diagnosis of PEN. Moreover, very rare chances of recuurence even after incomplete excision points in favour of reactive and benign nature of this tumour.

\section{Consent}

The examination of the patient was conducted according to the Declaration of Helsinki principles.

\section{ACKNOWLEDGEMENTS}

The authors sincerely thank Dr. Uday Khopkar for their contribution by giving valuable suggestions for the histopathological findings.

\section{REFERENCES}

1. Reed R, Fine R, Meltzer H. Palisaded encapsulated neuromas of the skin. Arch Dermatol. 1972;106:865-70.

2. Halder C, Sen S, Gangopadhyay A, Bala S. Zosteriform palisaded encapsulated neuroma: An unusual presentation. Indian J Dermatol. 2013;58:492.

3. Navarro M, Vilata J, Requena C, Aliaga A. Palisaded encapsulated neuroma (solitary circumscribed neuroma) of the glans penis. $\mathrm{Br}$ J Dermatol. 2000;142:1061-2.

4. Golod O, Soriano T, Craft N. Palisaded encapsulated neuroma-A classic presentation of a commonly misdiagnosed neural tumor. J Drugs Dermatol. 2005;4:92-4.

5. Zelger B, Steiner H, Kutzner H, Rutten A, Zelger B. Verocay body-prominent cutaneous schwannoma. Am J Dermatopathol. 1997;19:242-9.

6. Dover J, From L, Lewis A. Palisaded encapsulated neuromas. A clinicopathologic study. Arch Dermatol. 1989;125:386-9.

7. Argenyi ZB. Immunohistochemical characterization of palisaded, encapsulated neuroma. J Cutan Pathol. 1990;17:329-35.

8. Dubovy SR, Clark BJ. Palisaded encapsulated neuroma (solitary circumscribed neuroma of skin) of the eyelid: Report of two cases and review of the literature. Br J Ophthalmol. 2001;85:949-51.

9. Koutlas IG, Scheithauer BW. Palisaded encapsulated ("solitary circumscribed") neuroma of the oral cavity: A review of 55 cases. Head Neck Pathol. 2010;4:15-26.

Copyright by Anup Kumar Tiwary, et al. This is an open access article distributed under the terms of the Creative Commons Attribution License, which permits unrestricted use, distribution, and reproduction in any medium, provided the original author and source are credited.

Source of Support: Nil, Conflict of Interest: None declared. 